*Department of Experimental Oncology, European Institute of Oncology, Via Ripamanti 435, 20141, Milan, Italy. ${ }^{\ddagger}$ Department of Biomolecular sciences and Biotechnology, University of Milan, Milan 20100, Italy.

¿Department of Medicine, University of Milan, Milan 20100, Italy. Correspondence to S.M. e-mail:saverio.minucci@ ifom-ieo-campus.it doi: $10.1038 /$ nrc 1779

\title{
Histone deacetylase inhibitors and the promise of epigenetic (and more) treatments for cancer
}

\section{Saverio Minucci*‡ and Pier Giuseppe Pelicci*§}

\begin{abstract}
Histone deacetylases (HDACs) are considered to be among the most promising targets in drug development for cancer therapy, and first-generation histone deacetylase inhibitors (HDACi) are currently being tested in phase I/II clinical trials. A wide-ranging knowledge of the role of HDACs in tumorigenesis, and of the action of $\mathrm{HDACi}$, has been achieved. However, several basic aspects are not yet fully understood. Investigating these aspects in the context of what we now understand about HDACi action both in vitro and in vivo will further improve the design of optimized clinical protocols.
\end{abstract}

Histone deacetylases (HDACs) have been intensively scrutinized over the past few years for two main reasons. First, they have been linked mechanistically to the pathogenesis of cancer, as well as several other diseases. Second, small-molecule HDAC inhibitors (HDACi) exist that have the capacity to interfere with HDAC activity and can therefore achieve significant biological effects in preclinical models of cancer. These findings justified the introduction of HDACi into clinical trials. These initial clinical trials have just ended and show encouraging results. At this stage it is crucial to evaluate whether our current knowledge on the mechanisms of tumorigenesis that are linked to HDACs, and on the mechanisms of tumour sensitivity to HDACi, is firm enough to improve the design of further clinical trials. Recent structural and chemical data have emerged that will help in the design of novel HDACi with more desirable properties than the existing ones. However, key areas of investigation that might help to further illuminate the design of successful HDACi-based cancer therapy remain poorly explored. Novel findings indicate that our understanding of how HDACi work will probably change significantly, establishing a new paradigm in the field of intelligent drug design with broad implications for the design of targeted therapies in cancer and possibly other diseases.

\section{HDACs: enzymes looking for substrate(s)}

Four HDAC classes have been identified (FIG. 1a). One of them (class 3 or the so-called sirtuins, from the yeast protein Sir2) constitutes a structurally unrelated, NADdependent subfamily, and will not be considered here; neither will the class 3-specific HDACi, which are less characterized than those for the other classes ${ }^{1}$.
An extensive phylogenetic analysis of HDACs has been performed ${ }^{2,3}$. HDACs are members of an ancient enzyme family found in animals, plants, fungi and bacteria. It is thought that HDACs evolved in the absence of histone proteins. Indeed, eukaryotic HDACs can deacetylate non-histone as well as histone substrates, and some HDACs reside in the cytoplasm (where histones are synthesized and acetylated for proper assembly, without the intervention of HDACs) and in mitochondria (where histones are absent).

Are HDACs, then, truly HDACs ${ }^{4}$ ? We postulate that key HDAC substrates might not be histones, but instead belong to the growing list of acetylated non-histone proteins (FIG. $1 \mathrm{~b}$ and Supplementary information S1 (table)). Additionally, non-protein molecules such as polyamines or metabolic intermediates might also serve as valid substrates (as found in bacteria).

The search for other substrates, however, should not undervalue the fact that histones (as a bulk mass) are by far the most abundant HDAC substrate, and that histone acetylation surely represents a key target for the action of most HDACs.

\section{The core of the code}

Histones and DNA constitute the nucleosomes, structural units of chromatin that are essential in packaging eukaryotic DNA. The tail region of the histones (which extend away from the nucleosome core) undergo a complex and coordinated series of regulatory modifications ${ }^{5-7}$. These modifications can also occur within the globular domain of histones that make extensive contacts with DNA (see FIG. 1c for a list of the known acetylation sites in histones). 


\section{At a glance}

- Histone deacetylases (HDACs) and histone acetylases (HATs) are enzymes that are responsible for deacetylating and acetylating, respectively, the amino-terminal tails of histones. These chromatin changes regulate transcription and many other nuclear events.

- Non-histone proteins (such as the oncosuppressor p53) and several cytoplasmic proteins are also regulated by HATs/HDACs.

- Studies on the molecular pathogenesis of acute myeloid leukaemias have shown that the aberrant recruitment of HDACs has an important role in leukaemogenesis.

- Leukaemia-associated fusion proteins (such as promyelocytic leukaemia (PML)retinoic acid receptor (RAR) and acute myeloid leukaemia 1 (AML1)-ETO) recruit HDACs to repress the transcription of genes involved in differentiation (the fusion proteins therefore block differentiation) and impair the function of p53.

- Alterations in the expression and/or activity of HATs/HDACs have been also observed in solid tumours. Solid tumours show decreased levels of histone acetylation, which correlates with clinical outcome.

- HDAC inhibitors (HDACi) have been widely studied and belong to several chemical classes.

- HDACi exert cell-type-specific effects inducing apoptosis, cell-cycle arrest, and differentiation.

- In leukaemias, HDACi induce the expression of members of the tumour-necrosis factor-related apoptosis-inducing ligand (TRAIL) and FAS death receptor pathways. This induction is responsible for the pro-apoptotic effects of HDACi.

- Clinical trials for several HDACi have started, and HDACi-responsive tumours have been observed.
Enhanceosome

The assembly of higher-order three-dimensional transcription

factor-enhancer DNA

complexes that are required to

activate a gene.
It might be oversimplistic to try to assign specific regulatory functions to defined post-translational modifications. The widely used, and in some cases misused, term 'histone code' emphasizes the fact that some chromatin functions are specified by multiple and coordinated histone modifications, and that the same posttranslational modification does not necessarily function in a similar manner in other contexts. However, this rule has many exceptions, to the extent that it seems more appropriate to consider the various post-synthesis modifications of histones as an epigenomic alphabet, in which each modification is a letter, and combined modifications at a defined genomic region constitute a word, which might have different functional meanings in different contexts. Recently, a histone alphabet has been proposed to unify the nomenclature of histone modifications and is based on similar principles ${ }^{8}$.

Acetylation (particularly of histone H3 and histone $\mathrm{H} 4$ tails) has almost invariably been linked to a chromatin state that is poised for transcription or that corresponds to actively transcribed genomic regions 9 . Therefore, histone acetylases (HATs) and HDACs (acting in the context of multisubunit complexes; BOX 1) have traditionally been linked to activation and repression mechanisms, respectively. Additionally, acetylation of core histones has been correlated with other genome functions, including chromatin assembly, DNA repair and recombination ${ }^{10,11}$. A crucial role has also been demonstrated for histone acetylation in imposing replication timing of specific genomic regions ${ }^{12}$. In turn, this might lead to large-scale changes in gene expression ${ }^{13}$. In all of these cases, a complex spatio-temporal interplay between HATs and HDACs takes place, which is essential for the correct execution of these events. Many more years of work will be required to further advance our understanding of the role of histone acetylation in the regulation of nuclear events and in the context of the other chromatin modifications.

\section{The rest of the acetylome: new roles for (H)DACs}

Our knowledge of the non-histone HDAC substrates is rapidly increasing, although it remains fragmented. As for histones, we should view acetylation as part of a complex set of post-translational modifications often working cooperatively to regulate the function of the modified protein(s).

Identifying the entire acetylome deserves a much more aggressive and systematic experimental strategy than has been used so far. A potential approach would be the use of pan-specific antibodies that are able to recognize acetylated lysines in any protein context for proteomic approaches, similar to those used to study other post-translational modifications ${ }^{14}$. These antibodies are available and should produce a dynamic map of the acetylome and its function ${ }^{15,16}$.

A growing list of acetylated proteins is currently available (see Supplementary information S1 (table)). As expected from the cellular localization of HATs and HDACs, both cytoplasmic and nuclear proteins can undergo reversible acetylation. Acetylation of a protein can have many different effects.

Effects on protein stability. Both acetylation and ubiquitylation often occur on the same amino-acid residue (lysine), and there is a direct cross-talk between these two modifications ${ }^{17}$. Competition between acetylation and ubiquitylation influences the stabililty of the substrate and, therefore, indirectly regulates its function. So, HDACs can decrease the half-life of several substrates by exposing the lysine residue for ubiquitylation ${ }^{18-21}$ (FIG. 1 b). In some cases, the cross-talk is more complex, with both direct and indirect effects (see below).

Effects on protein-protein interactions. In the case of the interferon- $\beta$ enhanceosome, different HATs acetylate different lysine residues of the structural protein high mobility group protein isoforms I and Y (HMGI/Y, also known as HMGA1) in a precise order. This confers either potentiation of transcriptional activity or destabilization of the enhanceosome and termination of the transcriptional response $\mathrm{e}^{22}$. In the case of the transcription factor signal transducer and activator of transcription 3 (STAT3), which is activated by cytokine signalling, cytosolic acetylation triggers STAT3 dimerization and subsequent nuclear translocation ${ }^{23,24}$. Acetylation of hypoxia-inducible factor 1 (HIF1) by the ARD1 HAT apparently leads to increased association with the von Hippel-Lindau (VHL) ubiquitylation complex and proteasome-mediated degradation, which has a regulatory role in the cellular response to changes in oxygen availability and angiogenesis ${ }^{25,26}$. Another process that is regulated by reversibile acetylation is the cytosolic association of the mainly nuclear DNAdamage-response protein Ku70 (also known as XRCC6) 
A
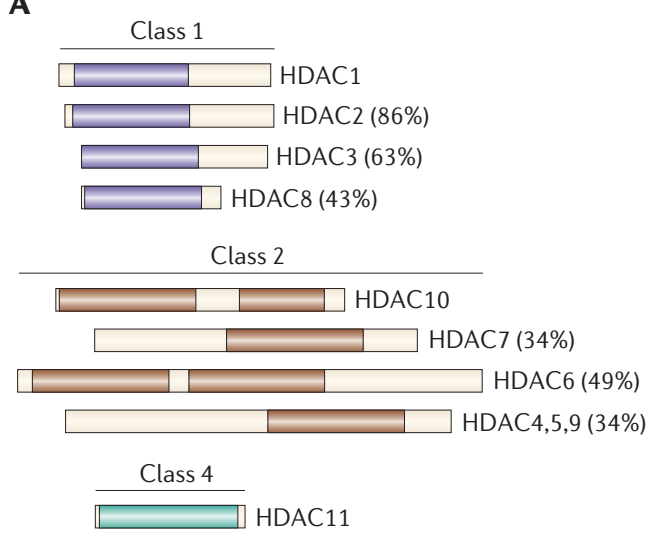

\section{C}

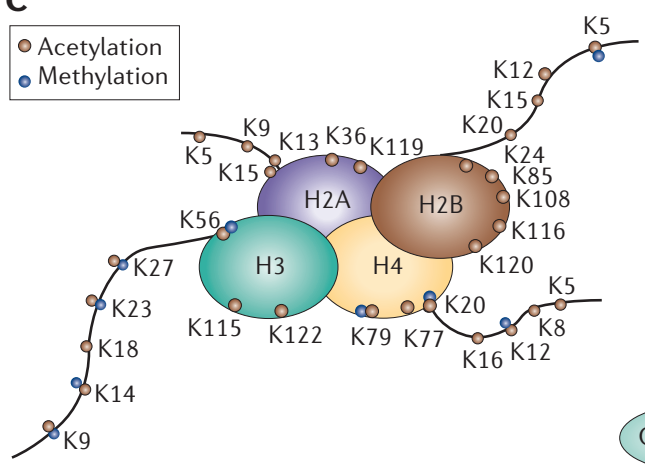

B

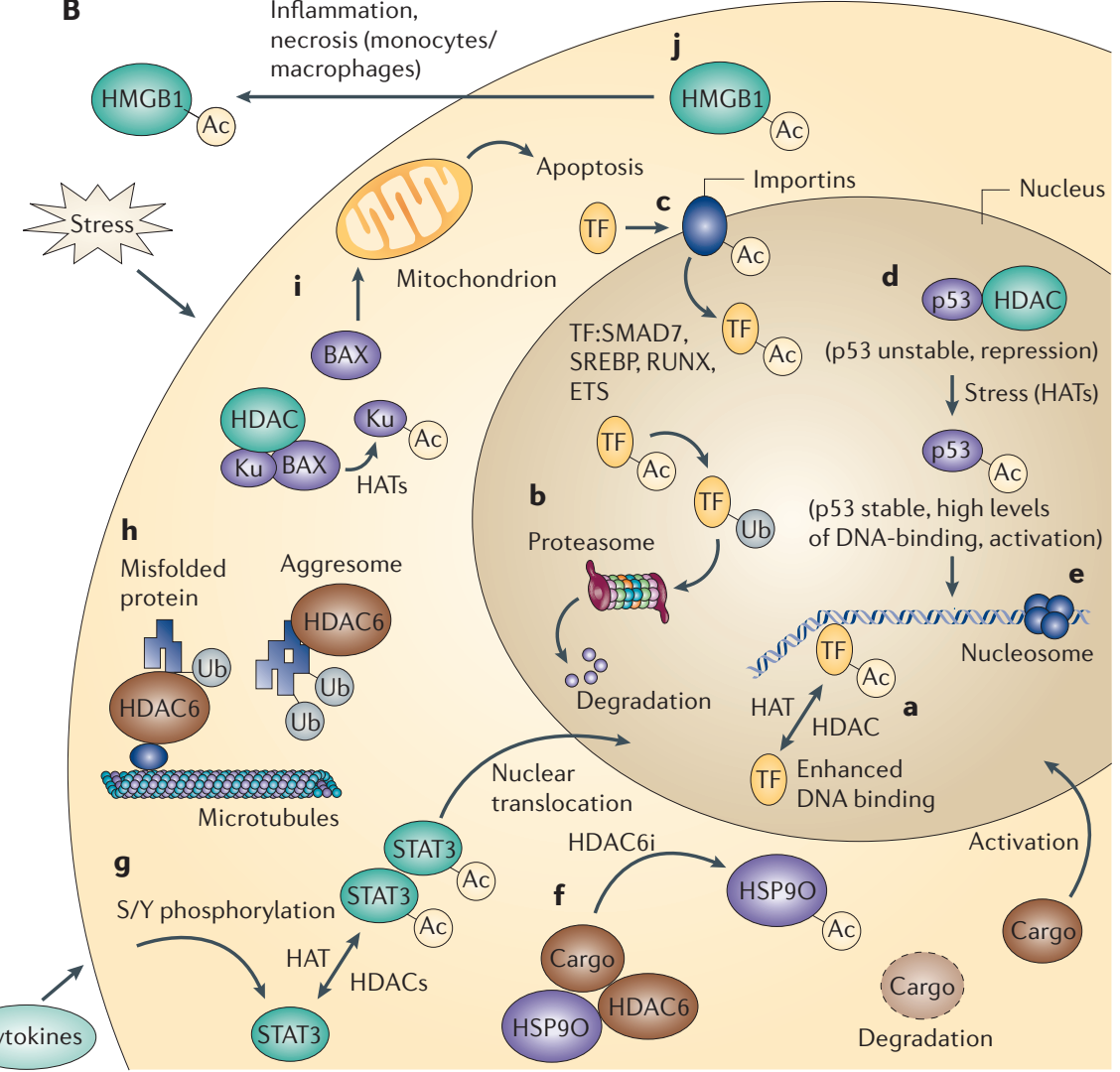

Figure 1 | The acetylome. A | A schematic representation of histone deacetylases (HDACs). Class 1, 2 and 4 human HDACs are represented, with the catalytic domains of the different classes in different colours. The degree of homology (entire sequences) between HDACs belonging to the same class are in brackets. All HDACs share a zinc-dependent catalytic domain with a high degree of homology. Less conserved are accessory domains, which seem to fulfill regulatory functions. In mammals, class 1 HDACs (HDAC1-3 and HDAC8) are related to the yeast RPD3 HDAC; class 2 HDACs (HDAC4-7, HDAC9 and HDAC10) are related to the yeast HDA1 HDAC, and have a more complex domain organization; a single HDAC, HDAC11, is the unique member of class 4 HDACs. Except for class 4, HDACs are found in all fully sequenced, free-living eukaryotic organisms. Class 4 proteins are found in all of these organisms except fungi. The conservation of these proteins indicates that each HDAC class has a non-redundant role in basic cell biological processes. Class $1 \mathrm{HDACs}$ are widely expressed, whereas classes 2 and 4 show various degrees of tissue specificity. In addition, class 1 and 4 HDACs are constitutively nuclear proteins, whereas class 2 HDACs shuttle between the nucleus and the cytoplasm owing to the reversible interaction with 14-3-3 proteins ${ }^{137}$. B $\mid$ A schematic view of the functioning acetylome. A partial list of biological processes that are regulated mechanistically by acetylation is sketched in a-j. Acetylation (Ac) might regulate the association of transcription factors (TF - for example, SMAD7, SRBEP, RUNX and ETS) with the DNA (a). Protein stability is also influenced by histone acetyltransferases (HATs) and HDACs, as lysines are subject to both acetylation and ubiquitylation (b). The import and export of proteins into the nucleus is regulated by the acetylation of the importin proteins at the nuclear envelope (c). Multiple, coordinated functions of $\mathrm{p} 53$ are known to be regulated by acetylation (d) and accessibility of the nucleosome is another HAT- and HDAC-regulated process (see below) (e). Chaperone functions of heat-shock protein 90 (HSP90) have also been shown to be effected by acetylation. Acetylation of HSP90 in the absence of HDAC6 prevents HSP90 interacting with its target protein (f). The acetylation of signal transducer and activator of transcription 3 (STAT3) induces protein dimerization and translocation to the nucleus (g). The function of the aggresome is regulated by acetylation. HDAC6 is

14-3-3 proteins

These proteins are a family of conserved regulatory molecules that are expressed in all eukaryotic cells. 14-3-3 proteins have the ability to bind a multitude of functionally diverse signalling proteins, including kinases, phosphatases and transmembrane receptors. Recently, class 2 histone deacetylases have also been shown to interact with

members of the 14-3-3 family. required to properly recruit ubiquitylated proteins to aggresomes, and inhibition of its activity leads to accumulation of polyubiquitylated proteins (h). The translocation of BAX to the mitochondria is influenced by acetylation of the DNAdamage associated protein Ku70. When Ku70 is acetylated, BAX is free to localize to the mitochondria (i). Acetylation also favours nuclear export and cytosolic accumulation of HMG box 1 (HMGB1) before secretion (j). C | The acetylation map of histones. A schematic view of the histone octamer, with the histone tails showing the possible acetylation sites (yellow circles) and methylation sites (blue circles) when this occurs on the same residue. Histone tails undergo a complex, coordinated series of post-translational modifications (phoshorylation, acetylation, methylation, ubiquitylation, ADP-ribosylation, deimination, and probably other post-translational modifications) that are required to regulate chromatin function(s) ${ }^{6,7}$. Two important mechanisms govern the significance of histone modifications. They can generate docking sites for interaction with additional proteins; bromo-, chromo- and SANT-domains have been shown to recognize specifically modified histones, and to mediate the recruitment of accessory regulatory factors that contain those domains. Also, modifications of histones might affect the condensation state and higher order chromatin structure, owing to their capacity to modulate the exposure of charge patches on the surface of the nucleosomes ${ }^{5-7}$. 


\section{Box 1 | Histone deacetylase complexes}

A number of histone deacetylase (HDAC)-associated factors and HDACcontaining complexes have been described for class 1 HDACs. HDAC1 and HDAC2 are mainly found associated in the same molecular complexes with Mi2/ NURD (nucleosome remodelling and histone deacetylase), SIN3A and Co-REST, whereas HDAC3 is found in a different complex (the nuclear co-receptor (NCOR)/ silencing mediator for retinoid and thyroid hormone receptors (SMRT) complex ${ }^{126-133}$. HDAC 6 has been found in cytoplasmic complexes with proteins involved in the ubiquitin signalling pathway ${ }^{134}$. Large multi-enzymatic complexes that contain histone acetyltransferase (HAT) or HDAC have also been described, which might represent the biochemical explanation of the dynamic nature of histone acetylation ${ }^{135}$. Accessory factors might crucially function by 'tethering' the complex to defined genomic regions (looking at chromatin as the main substrate), and to integrate modulatory signals through interactions with other proteins. Apparently, HDACs of different classes can co-exist in the same macromolecular complex. It has been suggested that the HDAC 3 complex maintains the enzymatic activity of class $2 \mathrm{HDACs}^{136}$. These studies have been performed by biochemical purification of native complexes or by immunopurification of tagged HDACs (or HDAC-associated factors). These studies were performed in cell lines and cannot be easily reproduced in normal cells, so it remains to be fully understood whether there are differences in the composition of the complexes according to the cell state. The different results reported by various groups might reflect the heterogeneity of the HDACcontaining complexes in different cells at different stages of differentiation.

with the pro-apoptotic protein BAX. In its deacetylated form (maintained by several HDACs), Ku70 keeps BAX away from the mitochondrion and protects cells from apoptosis. When the cell is stressed, HATs acetylate Ku70, leading to dissociation of the complex and translocation of BAX to the mitochondria, where it activates the apoptotic programme $e^{27,28}$.

Effects on protein localization. As already described for some factors (STAT3 and BAX), reversibile acetylation affects the subcellular localization of several proteins. In some cases, the nuclear localization signal contains acetylatable lysine residues that favour nuclear retention when acetylated ${ }^{23}$. In the case of the multifunctional HMG box 1 (HMGB1) protein, acetylation favours nuclear export and cytosolic accumulation before secretion during inflammatory and/or necrotic processes ${ }^{29}$. Proteins involved in nuclear import can themselves be regulated by acetylation ${ }^{30}$.

Effects on DNA binding. Several transcription factors show increased DNA binding and subsequent transcriptional activity when acetylated, which correlates with the hyper-acetylation of histones in target chromatin (see references in Supplementary information S1 (table)). Perhaps more surprisingly, in some cases acetylation impairs the binding of transcriptional activators to the DNA, indicating that HATs and HDACs might work in an orchestrated way to achieve the same cellular effect ${ }^{31}$. Interestingly, general transcription factor 2B (GTF2B, also known as TFIIB) has been reported to behave as an auto-acetyltransferase, and acetylation regulates its activity ${ }^{32}$. Acetylation also impairs the catalytic and DNA-binding activities of enzymes involved in DNA metabolism and repair $^{33,34}$.
Other effects. In some cases, the function of HAT/ HDAC substrates is influenced by acetylation at several levels. The oncosuppressor p53 is regulated by acetylation, which regulates the stability of the protein, its interaction with DNA and its transcriptional activity ${ }^{35}$. Similarly, the DNA-binding affinity of the transcription factor nuclear factor $\kappa \mathrm{B}(\mathrm{NF}-\kappa \mathrm{B})$ (in its multiple forms) is regulated by HATs/HDACs, as is its transcriptional activation and its association with its regulator, inhibitor of $\kappa \mathrm{B}-\alpha(\mathrm{I} \kappa \mathrm{B} \alpha)^{36}$. Cytoplasmic HDAC6 is crucial for the formation of aggresomes (organelles required for the efficient clearance of misfolded, toxic, cytoplasmic proteins), although in this case the role of protein acetylation remains unclear ${ }^{37}$. Recently, HDAC6 has been shown to deacetylate the cytoplasmic chaperone protein heat-shock protein 90 (HSP90). HSP90 hyperacetylation in HDAC6-deficient cells leads to the loss of chaperone activity ${ }^{38}$.

Given the multitude of effects that HDACs have, deciphering the phenotypes of HDAC-deficient model organisms is not always easy. Among class 1 HDACs, only HDAC1 has been knocked out in mammals so far. The phenotype is embryonic lethal at very early stages of development, mainly owing to an arrest of cell growth that is associated with upregulation of the cyclin-dependent-kinase (CDK) inhibitors p21 (also known as WAF1) and p27 (also known as KIP1). This shows a crucial, non-redundant role for HDAC1 in regulating cell proliferation ${ }^{39}$. It is unknown whether the other class 1 HDACs behave similarly. Consistent with their more restricted pattern of expression, class 2 HDAC-knockout mice have demonstrated a crucial role for distinct class 2 HDACs in modulating the growth response of specific tissues such as cartilage and heart muscle. In these cases, association of class 2 HDACs (HDAC5 and HDAC9 in cardiomiocytes and HDAC4 in chondrocytes) with specific transcription factors (MEF2 in heart and RUNX2 in cartilage) leads to repression of genes that are involved in cell growth. Accordingly, the corresponding knockouts show - in contrast to what is observed in Hdac1-null mice - hypertrophic growth $^{40,41}$. The analysis of HDAC-knockout mice has just started, and we do not precisely understand the cellular responses to the knockout of specific HDACs and how they are dependent on changes in acetylation of histones or of other HDAC substrates. In the case of $\mathrm{Hdacl}^{-/-}$cells, a general increase in histone acetylation has been observed, pointing to HDAC1 as a crucial component in the control of acetylation. The generation of conditional knockout strains, or the systematic use of RNA interference (RNAi) to knockdown HDAC expression in specific tissues after embryonic development, would also be helpful. Unfortunately, the available HDAC-knockout models have so far not been useful in addressing tumour susceptibility.

\section{HDACs in cancer: a matter of balance}

We can expect both positive and negative effects of HDACs on oncogenic and oncosuppressive mechanisms. A balance must exist, and shifts in this balance might have dramatic consequences on the cell phenotype. 
The most informative evidence on how this balance is altered in cancer cells comes from studies on the pathogenesis of leukaemias ${ }^{42}$.

Acute promyelocytic leukaemia (APL) was the first model disease in which the involvement of HDACs was demonstrated ${ }^{42}$. This form of leukaemia is characterized by an arrest of the leukaemic cells at the promyelocytic stage of myeloid differentiation, and is caused (as shown in murine models of the disease) by fusion proteins (found in $100 \%$ of patients with APL) of retinoic acid receptor- $\alpha$ (RAR) with one of the following proteins: (in $>95 \%$ of cases) promyelocytic leukaemia (PML), ( $\approx 5 \%$ of cases) promyelocytic leukaemia zinc finger (PLZF) or (sporadically) other genes. Clinically, patients with APL (with the exception of patients expressing PLZF-RAR) respond to treatment with pharmacological doses of the RAR ligand, retinoic acid (RA). At the cellular level, the clinical remission is due to the re-initiation of the differentiation programme of the leukaemic cells, which go on to full neutrophilic differentiation and then die, physiologically, by apoptosis ${ }^{43}$.

RAR functions as a transcription factor. In the absence of RA, RAR is found at DNA response elements of RA-regulated genes and is associated with HDACcontaining complexes, and this contributes to the transcriptional silencing of these genes. At physiological concentrations of RA, a conformational switch leads to the release of the HDAC-containing complexes and to the association of RAR with transcriptional co-activators (including HATs) and subsequent transcriptional activation. RA target genes are involved in key cellular processes and RAR works in cooperation with several other transcription factors (for example, cytokine-regulated factors such as Stats) ${ }^{44}$ to initate transcription.

In APL, all fusion proteins maintain the capacity to bind to RA-regulated genes, but share an aberrant feature - physiological concentrations of RA are unable to trigger the switch, and HDACs remain associated to RA targets ${ }^{45}$. To make things worse, RAR fusions oligomerize through self-association domains (present in PML, PLZF and the other genes), which leads to an increased stoichiometric association of HDACcontaining complexes with RA target genes and increased transcriptional silencing ${ }^{46,47}$. Additionally, recruitment by the fusion protein of other proteins with chromatin modifying activities (DNA methyltransferases and histone methyl-transferases; FIG. 2a) packs the chromatin to an extent that does not allow other physiological stimuli acting on the same genes (that is, cytokines that normally trigger differentiation) to function properly ${ }^{48,49}$. Although not fully validated experimentally, this model explains most of the observed data. Most importantly, this model fits perfectly with the phenomenon of RA sensitivity (and RA resistance in the case of PLZF-RAR) of patients with APL. In fact, at pharmacological doses of RA (10 to 100 -fold higher than physiological doses) the switch is turned on, HDACs dissociate from RAR and the turnover of the fusion proteins (through proteasomal degradation) is significantly increased. These molecular events lead to the powerful differentiative burst of promyelocytes that is seen in treated patients. PLZF-RAR does not respond to RA, even at pharmacological doses, explaining the resistance of patients to RA. However, the combination of RA with HDACi is extremely effective in reactivating RA target genes and triggers a cellular response in vitro and in vivo in PLZF-RAR-positive APL patients ${ }^{50}$.

The multiple-hit model hypothesizes that the fusion protein has leukaemogenic potential, but needs to cooperate with one or more additional genetic mutations to trigger the development of leukaemia ${ }^{51,52}$. The search for these second hits is on, and obvious candidates have been carefully scrutinized. Among these, TP53 mutations are exceedingly rare in patients with $\mathrm{APL}^{53}$, but $\mathrm{p} 53$ function is compromised through other mechanisms. PML-RAR associates with p53 and along with class 1 HDACs causes its deacetylation and subsequent degradation through the MDM2-proteasome pathway in haematopoietic precursors. These results provide the first link between alterations in p53 acetylation and tumorigenesis, highlighting the relevance of the study of post-translational modifications of non-histone proteins in cancer ${ }^{54,55}$.

Fusion proteins involved in other forms of leukaemia share the capacity to abnormally recruit HDACcontaining complexes ${ }^{42}$. Of course, beyond the apparent similarities, differences do exist. In B-cell lymphomas for example, the oncogene BCL6 encodes a transcriptional repressor that requires HDAC recruitment for its oncogenic properties. Interestingly, BCL6 is negatively regulated through direct acetylation by p300 HAT, and acetylation disrupts its ability to recruit HDACs and to transform cells ${ }^{56}$.

\section{HDACs and HATs in solid tumours}

In solid tumours, there is patchy but significant evidence for a disruption in the balance of acetylation and deacetylation (FIG. 2b). Specific HATs (p300 and CREB (cAMP response element-binding protein)-binding protein (CBP)) are targets of viral oncoproteins (adenoviral E1A, human papilloma virus E6 and the simian virus 40 (SV40) large T antigen) ${ }^{57}$. Monoallelic mutations of both p300 and CBP are found in patients who are affected by the congenital Rubinstein-Taybi syndrome - patients with this syndrome show developmental defects and are prone to cancer $^{58}$. Mutations and inactivations of specific HATs have been observed in a small number of patients with cancer. As for leukaemias, comparable mutations have so far not been observed for HDACs, hinting at the selection of a hyperactive HDAC phenotype in cancer cells ${ }^{58}$. Intriguingly, hyper-expression of HDACassociated factors occurs relatively often; genes of the metastasis-associated protein (Mta) family (in particular, MTA1), are associated with the metastatic phenotype in several tumour types ${ }^{59}$. The mechanistic consequences of overexpressing MTA1 are not well understood. MTA1 associates with the oestrogen receptor and inhibits its function, and in transgenic mice it induces alterations in mammary gland development that lead to tumour formation $^{60}$. MTA1 and other genes are thought to have a crucial function in integrating the enzymatic activity 
of HDACs with cellular signalling pathways. Therefore, their deregulated activity might also lead to a change in the activity of the HDACs. However, this has not been experimentally observed so far. HDAC2 has been shown to interact functionally with the Wnt pathway, as it is overexpressed in tumours and tissues from mice that lack
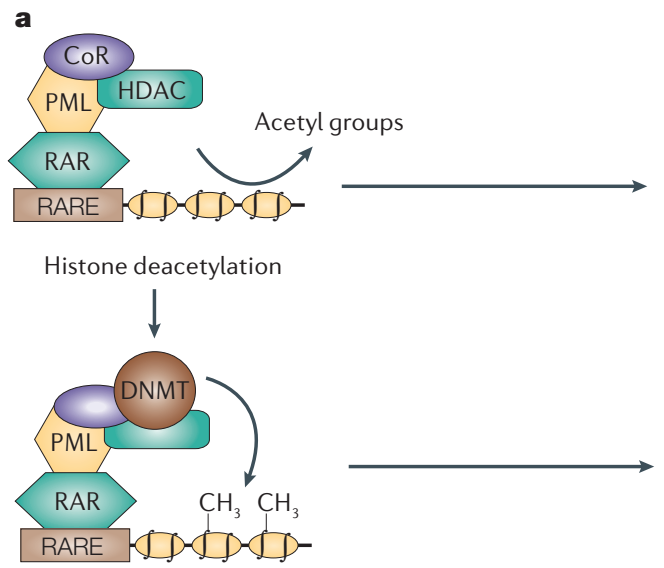

DNA methylation

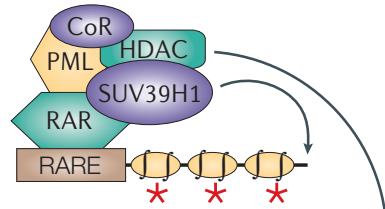

Histone methylation

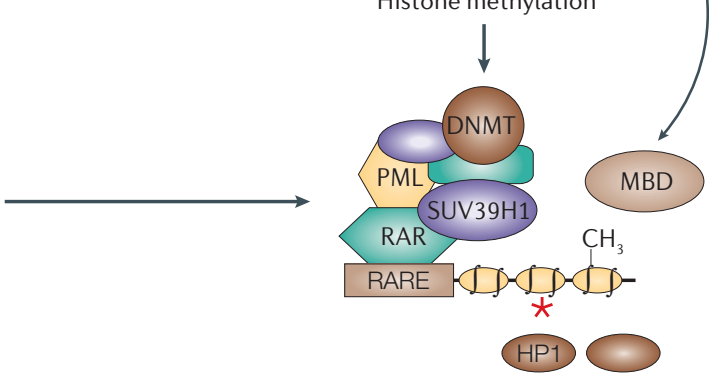

Heterochromatin-like formation

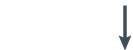

Differentiation block

b
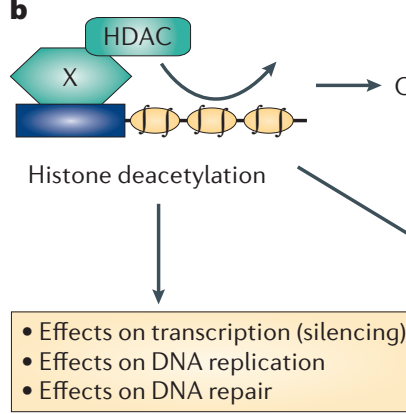

Figure 2 | A model for the deregulated action of HDACs on chromatin in APL and in other cancer cells. a | In acute promyelocytic leukaemia (APL), retinoic acid receptor (RAR) fusion proteins (represented by promyelocytic leukaemia (PML)-RAR in the scheme) recruit histone deacetylases (HDACs) (through co-regulatory (CoR) proteins such as nuclear co-receptor (NCOR)/silencing mediator for retinoid and thyroid hormone receptors (SMRT)) to the promoters of genes containing retinoic acid-responsive elements (RARE), leading to the deacetylation of histones. PML-RAR will then sequentially recruit other chromatin modifiers (DNA methyltransferases DNMT1 and DNMT3a, and the histone $\mathrm{H} 3 \mathrm{~K}$ 9-methyltransferases SUV39H1 and SUV39H2) that will methylate DNA $\left(\mathrm{CH}_{3}\right)$ and histones (asterisks). The modified chromatin functions as a docking site for accessory factors that are able to bind methylated DNA (methyl-CpG-binding domain proteins such as MBD1) and methylated histones (such as the heterochromatin associated HP1 protein), thereby leading to the formation of a compact 'heterochromatin-like' structure. Genes that are downregulated by PML-RAR are involved in myeloid differentiation, and because of the action of the fusion protein they become refractory to physiological differentiating stimuli (for example, cytokines and hormones), resulting in the block of differentiation and prolonged proliferation. $\mathbf{b}$ | In non-APL cells, other factors (for example, in leukaemias: other fusion proteins such as acute myeloid leukaemia 1(AML1)-ETO; in lymphomas: BCL6; and in solid tumours: deregulated transcription factors) substitute for PML-RAR in targeting HDACs to specific genomic regions, possibly leading to the same or similar set of chromatin alterations observed in APL. The newly formed chromatin structure (directly, or through recruitment of accessory factors) determines an altered pattern of transcription (for coding areas), or of DNA replication or repair, which cooperate in the transformed phenotype. the adenomatosis polyposis coli (APC) tumour suppressor. RNAi-mediated knockdown of HDAC2 in colonic cancer cells resulted in cell death, indicating a role for HDAC2 in protecting cancer cells against apoptosis ${ }^{61}$.

Recently, attempts have been made to correlate altered levels and/or activity of HATs or HDACs with distinct epigenetic states of chromatin. Overexpression of MTA1 in oesophageal squamous cell carcinoma correlates with poor prognosis and lower levels of acetylated histone $\mathrm{H} 4$ in cancer cells ${ }^{62}$. A more comprehensive analysis of a panel of normal tissues, cancer cell lines and primary tumours has shown a widespread loss of monoacetylated (at K16) and trimethylated (at K20) forms of histone H4 in a large number of tumours ${ }^{63}$. This loss occurs in repetitive DNA sequences and parallels the global loss of DNA methylation in these areas, which is also associated with tumorigenesis ${ }^{63}$. Strikingly, there are clinical correlations between distinct histone modification patterns (modifications examined: histone $\mathrm{H} 3$ acetylation at $\mathrm{K} 9$ and $\mathrm{K} 18$, dimethylation of $\mathrm{K} 4$; and histone $\mathrm{H} 4$ acetylation at $\mathrm{K} 12$, dimethylation at R3) and risk of tumour recurrence in patients with low-grade prostate cancer ${ }^{64}$. Low levels of histone acetylation, which by themselves are not sufficient to draw statistically significant predictions, are correlated with a poorer clinical outcome. Needless to say, a compelling question is whether these changes are restricted to histones or are shared by other HDAC substrates.

Besides histone acetylation, alterations in other epigenetic pathways (not discussed here) clearly have an important role in tumorigenesis. Indirectly, the observation that alterations of other chromatin modifiers can be observed in tumours is an additional link to deregulation in the balance of histone acetylation - both DNA and histone methylation are tightly linked to repressive chromatin states and at least partially dependent on the concomitant action of HDACs ${ }^{65-67}$. To date, however, the most convincing evidence that HDACs behave differently in cancer cells than in normal cells derives from the pharmacological manipulation of HDACs through HDACi.

\section{Inhibiting HDACs}

A dogmatic view of target identification for drug development would indicate that HDACs are not suitable targets - inhibiting HDACs has too many chances of interfering with key cellular functions. Luckily, the use of HDAC inhibitors pre-dated the discovery of the HDACs themselves. In fact, the inhibitors were instrumental in the purification and cloning of mammalian HDACs.

A relatively wide range of structures have been identified that are able to inhibit the activity of class 1 , class 2 and class $4 \mathrm{HDACs}^{68,69}$. They derive from both natural sources (FIG. 3a) and from synthetic routes (FIG. 3b). With a few exceptions, they can all be divided into chemical classes including hydroxamic acid derivatives, carboxylates, benzamides, electrophilic ketones and cyclic peptides $^{70,71}$. So far, these inhibitors work equally well against class 1, class 2 and class 4 HDACs. Only a few molecules are emerging as preferential inhibitors of class 1 versus 
a HDAC inhibitors from natural sources

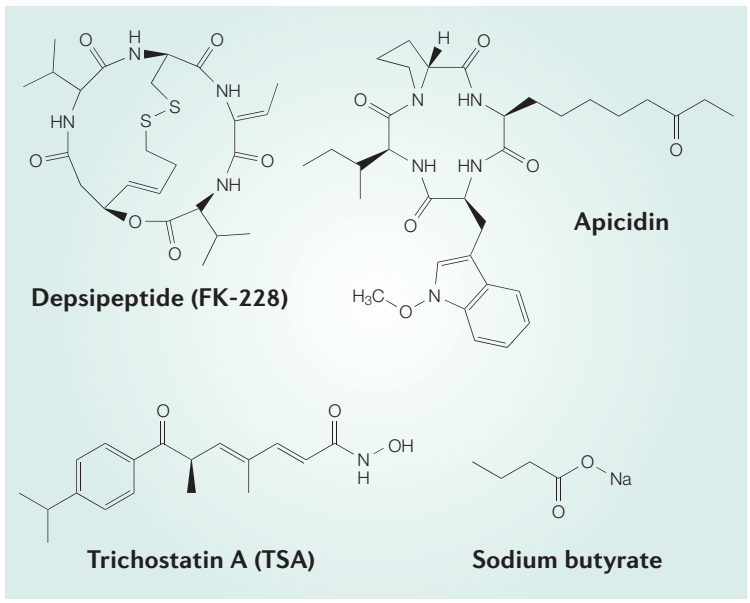

C Pharmacophore model for HDAC inhibition
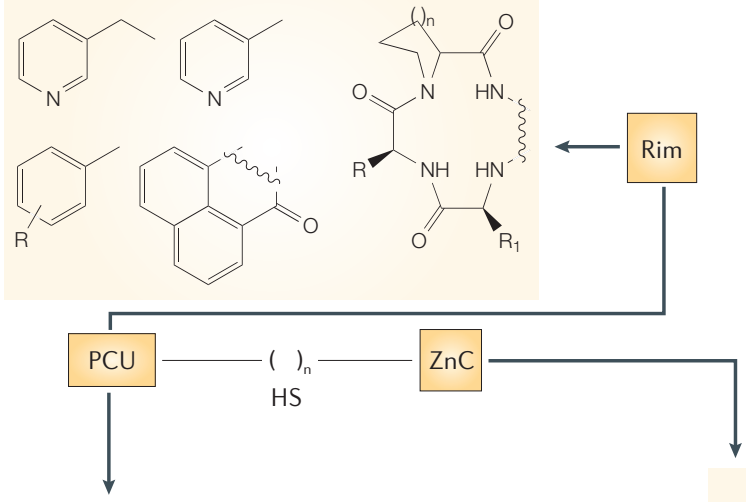

$\mathrm{H}_{\mathrm{NH}}^{\mathrm{O}} \mathrm{H}_{\mathrm{O}}^{\mathrm{O}} \mathrm{H}_{\mathrm{H}}^{\mathrm{O}}$ b Synthetic HDAC inhibitors

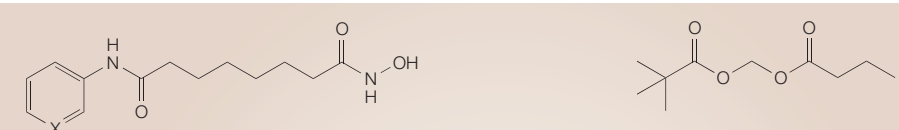

$\mathrm{X}=\mathrm{CH}$, Suberoyl anilide hydroxamic acid (SAHA) AN-9 (Pivanex) $\mathrm{X}=\mathrm{N}$, Pyroxamide
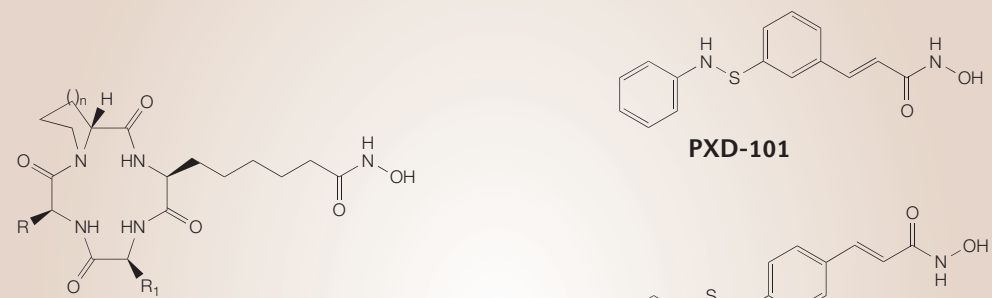

Cyclic hydroxamic acid-containing peptides (CHAPs) R = Benzyl, methyl, sec-butyl

$\mathrm{O}=$ Benzyl, 4-methyl-benzyl

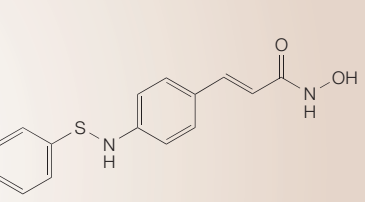

Sulfonamide hydroxamic acid

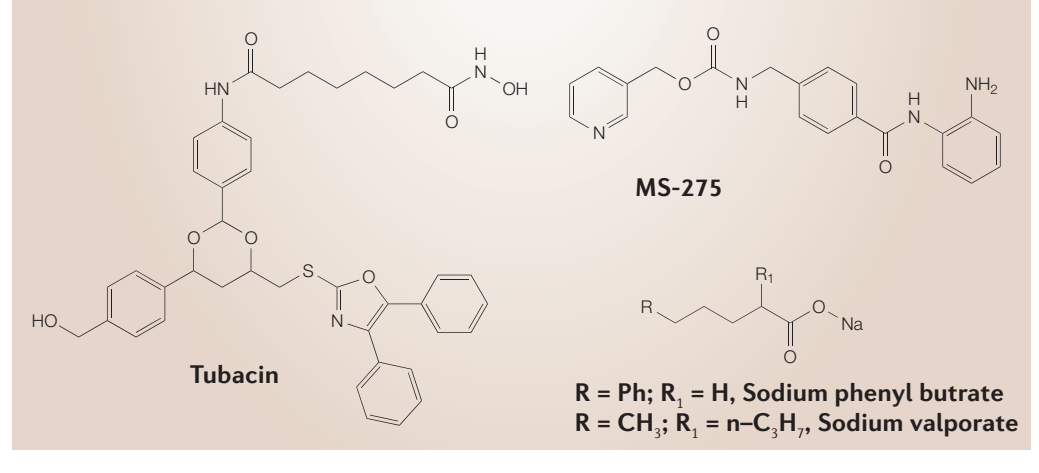

Figure 3 | HDAC inhibitors. Representative structures of the main classes of known histone deacteylase (HDAC) inhibitors (HDACi), either from natural sources (a) or synthetic (b). In HDACs, the active site of the enzyme (containing the zinc atom) occupies the bottom of a channel delimited by a rim, which corresponds to the substrate pocket (acetyl-lysine). The schematic view of the HDACi 'pharmacophore' (c) therefore consists of: a metal-binding domain $(\mathrm{ZnC})$, which chelates zinc and blocks the enzymatic activity; a linker domain, which mimics the substrate and occupies the enzymatic channel (PCU and HS: polar connecting unit and hydrophobic spacer, respectively); and a surface domain, which makes contacts with the im $^{71}$. The structures of the HDACi chemical groups that carry out these processes are shown below each of the yellow boxes, as indicated by the arrows.

class 2 HDACs, and even fewer (that is, tubacin for HDAC6) are able to discriminate efficiently among HDACs that belong to the same class ${ }^{72-75}$. At present, this limitation has little relevance to the use of HDACi as potential anti-tumour drugs as there is no definitive evidence that distinct HDACs have a defined role in cancer. On the other hand, HDAC-selective inhibitors would constitute (in addition to genetic tools) a useful experimental tool to address the issue of assigning distinct biological functions to individual HDACs.

From the structure of HDAC8 or HDLP (a HDAClike protein isolated from Aquifex aeolicus), and the structure of these enzymes in complex with a small number of HDACi, a few key points have emerged about the active site that support a pharmacophore model for HDAC inhibition ${ }^{76-79}$ (FIG. 3c). For the purpose of this review, we will consider - unless specifically mentioned in the text - HDACi as a single entity as they display comparable biological activities. However, they might differ substantially in their pharmacological properties (potency, efficacy, stability and toxicity), and will therefore probably be subject to different degrees of drug development in the future.

\section{The cellular effects of HDACi}

HDACi induce, to a variable extent, growth arrest, differentiation or apoptosis in vitro and in vivo ${ }^{68,69}$. In some cases, growth arrest is induced at low doses, and apoptosis is induced at higher doses; in other cases, growth arrest precedes apoptosis. However, cells might undergo apoptosis without significant changes in their cell-cycle profile. Strikingly, normal cells 
are almost always considerably more resistant than tumour cells to $\mathrm{HDACi}^{68}$.

It is worth stressing that there might be HDACidependent pathways that work against the desired anti-proliferation, pro-apoptotic and pro-differentiation effects of HDACi. One such example is the E2F family of transcription factors that regulate target genes that are crucial for S-phase progression. The oncosuppressor retinoblastoma $(\mathrm{RB})$ recruits HDACs to E2F target genes, thereby controlling G1/S cell-cycle progression. HDACi should therefore lead to activation of RB targets, effectively enhancing a key proliferative pathway. Despite these reservations, HDAC recruitment seems crucial only for a subset of RB targets, and cell-cycle regulation by $\mathrm{RB}$ proceeds largely through HDACindependent mechanisms ${ }^{80}$.

Given the known function of histone acetylation in transcription, it seems logical to postulate that inhibition of HDACs alone is unlikely to lead to a generalized increase in the transcription of all known genes. In fact, acetylation works together with other posttranslational modifications, and blocking deacetylation might have very different outcomes depending on the previous chromatin state. Up to $20 \%$ of all known genes are affected by $\mathrm{HDACi}^{81-84}$. Not all of these genes are necessarily upregulated by treatment - the ratio of upregulated to downregulated genes is close to $1: 1$.

Looking at chromatin as the target for HDACi action, we should not disregard the possibility that HDACi influence other nuclear phenomena besides transcription: what we have previously discussed indicates effects on DNA replication and repair, and both processes can have a key role in modulating the cellular response to HDACi. In fact, HDACi can inhibit DNA repair responses in a few cell lines, which might increase the sensitivity of tumour cells to chemotherapy and radiotherapy by leading to increased DNA damage by these treatment ${ }^{85,86}$.

At least in part, the failure to activate two cell-cycle checkpoints that are present in normal cells is responsible for the tumour-selective action of $\mathrm{HDACi}^{87}$. The first is the G2-phase checkpoint, which when defective permits cells to enter an aberrant mitosis. The second is the mitotic-spindle checkpoint, which normally detects aberrant mitosis and blocks mitotic exit until the defect is rectified. The disruption of both checkpoints results in the premature exit of tumour cells from an abortive mitosis and the subsequent induction of apoptosis ${ }^{88,89}$. One model of action for HDACi derived from these studies, and those on the effects of HDACi on DNA repair, is that HDACi should lead to an increased accumulation of DNA damage (by endogenous stress or by drug treatment) in sensitive cells. As the DNA in these cells will be unrepaired and these defects will remain unchecked owing to the altered cell-cycle checkpoints, this will lead to catastrophic cell death.

Given the pleiotropic effects of HDACs on nonhistone substrates, we must expect additional targets. These targets might be transcription factors themselves, and therefore the ultimate endpoint will eventually be transcriptional (which might help to explain why the transcriptional response to HDACi is so complex). It would be somewhat difficult to ascribe a prominent role to hyper-acetylation of chromatin or transcription factors in the observed response, as these modifications occur concomitantly. A partial dissection of the molecular mechanisms, however, is sometimes possibile. As an example, p21 (see below) is consistently upregulated by $\mathrm{HDACi}^{90}$. One of the key transcriptional regulators of $\mathrm{p} 21$ is p53. Strikingly, both hyper-acetylation of chromatin at the CDKN1A (which encodes $\mathrm{p} 21$ ) promoter and transcriptional activation of CDKN1A occur at the same level in wild-type and p53-null cells, implying that hyper-acetylation of p53 is not important for the therapeutic efficacy of HDACi (although acetylation of other transcription factors cannot be excluded $)^{91,92}$. HDACi-mediated acetylation of other substrates might lead to transcriptional effects only indirectly, or work through distinct, nontranscriptional mechanisms. In the end, HDACi might be considered as partially epigenetic drugs.

A complex pattern of HDACi targets must therefore exist that is cell-type-specific, cell-stage-specific, and dependent on the normal or pathological state of the cell. However, some general HDACi-mediated responses are known, and their relevance to the observed cellular effects can be studied. HDACi-induced growth arrest is tightly linked to the induction of p21, and in some cases this arrest has been shown to be irreversible and to resemble the phenomenon of replicative senescence $^{90,91,93}$. On the other hand, HDACi also induce cell death through caspase-dependent and caspaseindependent pathways. In most of the studies, caspase activation has been reported to occur through the mitochondria/cytochrome $c$-mediated apoptotic pathway. Nevertheless, HDACi can induce cell death with morphological features of autophagy in the absence of activated caspases, indicating that HDACi might work in cells with apoptotic defects ${ }^{94}$.

In HDACi-induced apoptosis, the mitochondrion and the production of pro-apoptotic reactive oxygen species (ROS) seems to be important ${ }^{94-96}$. The precise mechanisms underlying the accumulation of ROS are under investigation and might help us to understand why normal cells are selectively resistant to HDACi treatment. Normal, but not transformed, human fibroblasts accumulate thioredoxin (TXN, also known as TRX), a natural ROS scavenger, after treatment with HDACi. Therefore, ROS accumulation might be neutralized in normal cells by TXN, whereas transformed cells die because they do not express TXN: indeed, knockdown of TXN in normal cells causes accumulation of ROS and increased cell death ${ }^{96}$. Interestingly, $\mathrm{Bax}^{-/-}$murine embryonic fibroblasts are also resistant to $\mathrm{HDACi}^{97}$. As discussed earlier, hyper-acetylation of cytoplasmic Ku70 causes the activation of BAX and apoptosis ${ }^{97}$. HDACi also cause hyper-acetylation of HSP90 and its inactivation, leading to the degradation of proteins that require the chaperone function of HSP90 (including some oncoproteins ${ }^{98,99}$ ). Moreover, selective inhibition of HDAC6 by tubacin leads to cell death through accumulation of ubiquitylated 


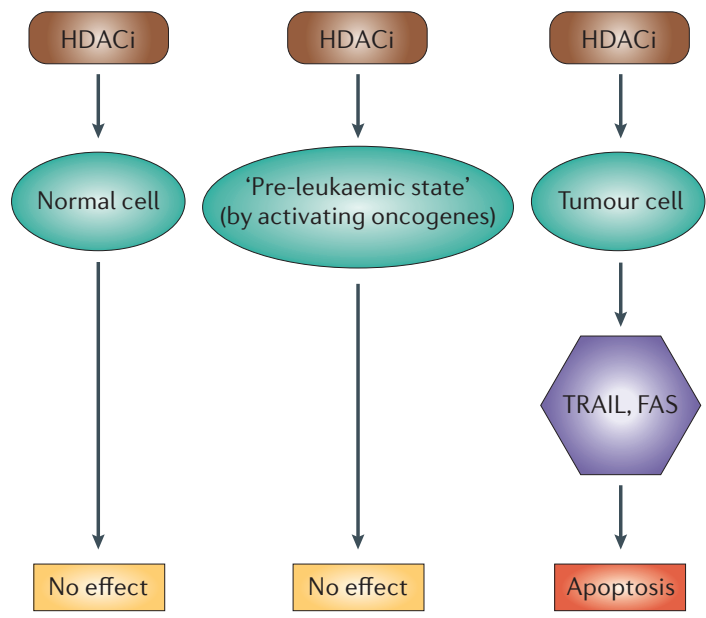

Figure 4 | Tumour-selective action of HDACi in acute promyelocytic leukaemia. Histone deacteylase (HDAC) inhibitors (HDACi) have little or no effect on short-term cultures of normal haematopoietic progenitors in vitro or on haematopoiesis in normal mice. Expression of the fusion oncoprotein promyelocytic leukaemia-retinoic acid receptor (PML-RAR) in haematopoietic progenitors leads to deregulation of differentiation and inhibition of $\mathrm{p} 53$ dependent stress responses ('pre-leukaemia'), but does not result in full transformation, which depends on further genomic hits. HDACi are not effective on cells at the preleukaemic stage, which behave similarly to normal cells. Following transformation, $\mathrm{HDACi}$ are able to induce members of the tumour-necrosis factor-related apoptosisinducing ligand (TRAIL) or FAS pathways, leading to tumour-specific cell death.

proteins $^{100}$. A limitation of these observations is their lack of systematic analysis, the use of cell lines as model systems - which do not necessarily reflect the biology of primary tumour cells - and the focus on a limited number of HDACi.

As a well-defined model system, APL offers an interesting paradigm for studying HDACi action (FIG. 4). HDACi trigger apoptosis of APL cells both in vitro and in murine models of the disease ${ }^{101,102}$. An obvious explanation for this effect would be the reversal of the HDAC-dependent action of the fusion protein, as discussed earlier in this review. Strikingly, however, HDACi function in APL through a fusion-protein-independent and p53independent mechanism (see below). But, in appropriate experimental conditions, such as on the induction of cellular stress, HDACi can revert the leukaemic cell phenotype through an effect on the RAR fusion proteins ${ }^{55}$. However, this effect is not necessary for the anticancer effects of HDACi.

\section{On the right TRAIL}

HDACi induce hyper-acetylation of the promoters of several members of the death receptor pathway including TRAIL (tumour-necrosis factor-related apoptosis-inducing ligand) and its receptor, death receptor 5 (DR5), and FAS ligand (FASL) and FAS, through a mechanism that seems to involve acetylation of the transcription factors SP1 or SP3, and subsequent recruitment of CBP. RNAi-based studies performed in vitro and in vivo show that these genes are uniquely required for the induction of apoptosis in leukaemic cells, both from APL mice and freshly isolated leukaemic blasts from patients who do not have APL. Intriguingly, not all blasts respond to HDACi by undergoing apoptosis, and these cases do not show induction of TRAIL or FAS pathways ${ }^{54,102}$. The reasons for this lack of response are unknown. Preliminary evidence indicates that HDACi might activate components of the death receptor pathways in various solid tumours. In fact, in several cases HDACi synergize with exogenously added TRAIL to induce apoptosis of tumour cell lines ${ }^{103-106}$. However, the effect on the death receptor pathway is not universal, and therefore its activation does not necessarily represent an obligate path for HDACi-mediated cell death.

In conclusion, many areas of investigation on the biological effects of HDACi show an exciting and complex network of cellular responses, coupling transcriptional and non-transcriptional effects. It is difficult at this stage to predict the existence of a universal, key cellular target that is responsible for the HDACi-mediated response. To try to address this question, it would be helpful to analyse systematically, and in different model systems, all of the proposed effectors of HDACi to define their relative contributions to the observed biological effects. In the absence of such studies, we can only speculate whether the different mechanisms that have been proposed represent cell-type-specific responses to HDACi, or whether the simultaneous activation of several pathways (or all of them required for maximal effect) have a synergistic function.

An encouraging observation is that in several cases normal cells show a strikingly reduced sensitivity to HDACi treatment, hinting at potentially large differences in the acetylome in normal versus tumour cells that can be exploited clinically.

\section{HDACi in clinical development}

The first studies on the clinical use of HDACi have been published recently ${ }^{107-109}$. These studies are phase I/II trials, and their endpoint is therefore somewhat limited (the main aim being to analyse/exclude unwanted toxicity and to find optimal doses/schedules), although one of the objectives of phase II studies is to measure the clinical response. It is not our scope to review in detail single studies on specific HDACi, but rather to draw initial and very preliminary conclusions, and to suggest how such trials need to evolve (TABLE 1).

These reports generally confirm the initial impression from preclinical studies of low toxicity of HDACi in patients, if compared with most of the currently used anti-tumour treatments. The toxic effects of HDACi, which vary based on the class of HDACi used, might at least in part be the result of the non-specific side effects of each individual HDACi rather than the consequences of inhibiting HDAC per se. In fact, some of these compounds are only efficacious at high concentrations in which interference with additional targets would probably be responsible for toxicity. As for most 
Table 1 | Summary of the main clinical studies using histone deacetylase inhibitors

\begin{tabular}{llll}
$\begin{array}{l}\text { Histone } \\
\text { deacetylase } \\
\text { inhibitor }\end{array}$ & Disease & Phase & Clinical results \\
\hline $\begin{array}{l}\text { AN-9 (butyric acid } \\
\text { prodrug) }\end{array}$ & $\begin{array}{l}\text { Lung cancer, melanoma } \\
\text { and leukaemias }\end{array}$ & $\mathrm{Ila} / \mathrm{llb}$ & $\begin{array}{l}\text { Monotherapy in lung cancer (non- } \\
\text { small-cell carcinomas); patients showed } \\
\text { partial responses in }<10 \% \text { of the cases }\end{array}$ \\
\hline
\end{tabular}

\begin{tabular}{|c|c|c|c|}
\hline $\begin{array}{l}\text { Sodium } \\
\text { phenylbutyrate }\end{array}$ & $\begin{array}{l}\text { Leukaemias and } \\
\text { myelodysplasia }\end{array}$ & I & $\begin{array}{l}\text { Haematological improvements with } \\
\text { no remissions; poor potency and } \\
\text { pharmacokinetic properties }\end{array}$ \\
\hline Valproic acid & $\begin{array}{l}\text { Leukaemias, } \\
\text { myelodysplasia and } \\
\text { cervical cancer }\end{array}$ & $\mathrm{I} / \mathrm{II}$ & $\begin{array}{l}\text { In combination with retinoic acid, } \\
\text { partial and complete remissions have } \\
\text { been observed in } 30 \% \text { of cases in a pilot } \\
\text { study, whereas an extended phase II } \\
\text { study showed only haematological } \\
\text { improvements }\end{array}$ \\
\hline $\begin{array}{l}\text { FK-228 (cyclic } \\
\text { depsipeptide) }\end{array}$ & $\begin{array}{l}\text { T-cell cutaneous or } \\
\text { peripheral lymphoma, } \\
\text { leukaemias and solid } \\
\text { tumours }\end{array}$ & $I / I I$ & $\begin{array}{l}\text { Monotherapy in T-cell lymphomas } \\
\text { showed partial and complete responses } \\
\text { in up to } 57 \% \text { of patients in a single } \\
\text { phase II study; one partial response was } \\
\text { observed in solid tumour patients }\end{array}$ \\
\hline $\begin{array}{l}\text { MS- } 275 \\
\text { (benzamide) }\end{array}$ & $\begin{array}{l}\text { Refractory solid } \\
\text { tumours, leukaemias } \\
\text { and lymphomas }\end{array}$ & I & $\begin{array}{l}\text { Long half-life in patients ( } 39-80 \\
\text { hours); no clinical responses have been } \\
\text { reported }\end{array}$ \\
\hline
\end{tabular}

Suberoylanilide hydroxamic acid (hydroxamic acid derivative)

\section{LAQ-824}

(hydroxamic acid derivative)

\author{
Refractory solid \\ tumours, leukaemias \\ and lymphomas
}

Side effects

Monotherapy was well

tolerated, whereas a

combination treatment with docetaxel resulted in severe toxicity and interruption of the study

Well tolerated, with neurological toxicity only at very high doses

Neurological toxicity

Bone marrow toxicity, reversible

cardiac arrhythmia (which seems not to be clinically relevant), fatigue, nausea and vomiting

Toxicity indicated that a once every 14 days oral schedule would be best; nausea, vomiting, anorexia and fatigure were the dose-limiting toxicities; cumulative bone marrow toxicity was also seen

Mild toxicity, dehydration, fatigue, diarrhoea and anorexia; thrombocytopaenia and anaemia were the doselimiting toxicities; no signs of cardiotoxicity

Bone marrow toxicity, fatigue, observed in leukaemias; a close analogue (LBH589) has also entered clinical studies

diarrhoea, nausea, and tachycardia (which is only observed following treatment with LBH589); signs of potential cardiac toxicity have been recently reported, which led to the interruption of the clinical development lack of haematological toxicities

The list is not fully comprehensive due to space limitations, but we have made an effort to include histone deacetylase inhibitors of different structures and to mention studies that showed clear clinical effects or serious toxic effects. NA, not available.

Maximal tolerated dose The maximal dosage of a particular drug that can be used before patients show undesired side effects. chemotherapic drugs, bone marrow toxicity (of low to moderate degreee) is often observed. Strikingly, in some cases the maximal tolerated dose or MTD has not been reached in clinical trials, implying that specific HDACi have minimal toxicity and a wide therapeutic window (TABLE 1).

Unfortunately, the relationship between the toxicity of HDACi and their pharmacodynamic/pharmacokinetic properties is still largely unknown. This makes it difficult to optimize HDACi treatment. In particular, we do not know which patients are most likely to respond to HDACi. Naively, we would predict that HDACi might be useful only in those tumours in which HDACs are directly involved in the pathogenesis. However, in light of the effects of HDACi in murine models of leukaemia, we anticipate that HDACi might be useful in other contexts as they might attack tumour-cell-specific targets that are not directly involved in tumorigenesis. We also do not know the key target(s) for HDACi action. Proposed surrogate markers, such as measuring the levels of acetylated histones from peripheral blood cells before and after treatment ${ }^{16}$, should serve as indicators of effectiveness, but these need to be validated clinically and do not always seem to correlate strictly with pharmacokinetic profiles ${ }^{110,111}$. Alternative strategies could be attempted to identify novel predictive markers; gene profiling studies 
from tumour samples obtained from responders and nonresponders might lead to their identification. Also, we do not know whether there is a requirement for continuous inhibition of HDACs to achieve the maximal effect.

Studies in preclinical models (such as leukaemias) have shown that an intraperitoneal injection of HDACi induces pulses of histone hyper-acetylation in tumour cells (lasting a few hours), followed by a return to basal levels. This response is owing to the fast degradation of the HDACi used in those studies, which resemble the kinetics and fast degradation of many HDACi (particularly those based on hydroxamic acid) in the human bloodstream. In mice, this treatment regimen leads to rapid and massive apoptosis of tumour cells, with little toxicity observed. Are we happy with this observation and to use it as a benchmark for a similar response in patients? Although a clinical response is observed in the mouse models (and in patients treated with HDACi - see below), animals are not cured, and as soon as the treatment is stopped, the disease kills them in $100 \%$ of the cases. It seems plausible that the continuous inhibition of HDAC activity will have a more positive long-term response, but will it also be more toxic for normal cells? Two different formulations of the same drug (oral and intravenous suberoylanilide hydroxamic acid (SAHA)) apparently confirm this trend. Oral SAHA has a more favourable pharmacokinetic profile (half-life of 120 minutes versus 40 minutes for intravenous SAHA) and results in a better clinical performance, but it is substantially more toxic $^{111,112}$. HDACi with substantially longer half-lives (such as MS-275, with a half-life of up to 80 hours) show a higher toxicity that precludes daily treatment ${ }^{113}$. Valproic acid is being taken at high doses by epileptic patients with no side effects, but this HDACi has an extremely low potency and most of the absorbed drug (up to $90 \%$ ) is bound to serum proteins, making it unlikely to significantly inhibit HDACs at the doses used in these patients. Notwithstanding these caveats, promising results have been observed in terms of clinical response in the current trials. None of the HDACi tested in clinical trials is completely without clinical effect, confirming that HDACs represent a promising target for further development. The results have been especially good in patients with T-cell cutaneous lymphoma, and in some cases a long-term response has been observed ${ }^{114,115}$. The molecular basis for this response is being explored in vitro ${ }^{116}$. Tumour regression (in some cases quite dramatic) has also been observed in solid tumours ${ }^{111,112}$.

Acquired resistance to HDACi has been reported anecdotally, implying that it might be a clinically rare event ${ }^{117}$. Depsipeptide has been reported to be a substrate for P-glycoprotein (Pgp, also known as ATP-binding cassette subfamily $\mathrm{B}$, member 1 ( $\mathrm{ABCB} 1)$ ), and multidrug resistance-associated protein 1 (MRP1, also known as $\mathrm{ABCC} 1$ ), and to induce Pgp expression, indicating that resistance to HDACi might be drug-specific ${ }^{116,118}$.

Systematic in vitro studies have explored the possibility that HDACi might synergize with other drugs ${ }^{109}$. The effect of a wide spectrum of chemotherapeutic drugs is potentiated by HDACi, but the mechanistic basis for this synergy has not been fully explored. Given the results previously discussed, one way to increase sensitivity to chemotherapy and/or radiotherapy would be the suppression of checkpoint and repair mechanisms triggered by induced DNA-damage. Unfortunately, synergism in efficacy might be accompanied by adverse effects that are rarely or never seen with HDACi alone, and caution should be employed when considering clinical studies that address HDACi in combination with other therapies. Clinical studies combining RA treatment with HDACi are in progress in patients with leukaemia, expecially in the elderly ${ }^{119}$. Of particular interest is the combination of HDACi with other drugs acting on epigenetic mechanisms. DNA-demethylating agents in combination with HDACi show potent responses in vitro and they are also being tested in clinical trials ${ }^{120}$. The combination of HDACi with DNA-demethylating agents should be more carefully analysed in murine models of tumours, with a view to optimizing the design of clinical studies. HDACi might also work synergistically with HSP90 inhibitors; this would further inhibit the capacity of HSP90 to chaperone oncoproteins that are required for the survival of tumour cells ${ }^{121}$.

\section{Perspectives}

The prevailing conclusion is one of excitement for our progress in understanding the function of HDACs in tumour pathogenesis and the tumour response to HDACi, and hope for the exploitation of this knowledge to develop more effective clinical protocols.

There are areas that we have not covered that might become relevant in the future. For instance, the regulation of HDAC enzymatic activity through post-translational modifications (phosphorylation or sumoylation) could turn out to be very important in modulating HDAC function. This could potentially be exploited pharmacologically ${ }^{122}$. Another issue to be considered is that, so far, there is no conclusive experimental evidence that points to specific HDACs as being selectively involved in any form of disease, including cancer. A systematic analysis to try to identify selective HDACi that will enable the study of specific HDACs is mandatory if we are to develop potentially less toxic and more effective treatments. There are other areas that we have discussed above that also require urgent answers.

A more general consideration also needs to be addressed. It has becoming increasingly clear that tumour cells are not homogeneous and that cancer stem cells exist $^{123,124}$. It is reasonable to assume that the failures of current cancer therapy are in part due to the failure to target cancer stem cells. HDACi affect the maintenance of stem-cell potential in $\mathrm{CD}_{3} 4^{+}$human haematopoietic progenitors in vitro ${ }^{125}$. So far, no study using HDACi (either preclinical or clinical) has addressed the crucial issue of tumour stem-cell sensitivity, which should be clarified as soon as possible to verify the true potential of HDACi in clinical treatment.

Perhaps, an epigenetic (or partially epigenetic) therapy for cancer (the 'epi-cure') is getting closer. 
1. Blander, G. \& Guarente, L. The Sir2 family of protein deacetylases. Annu. Rev. Biochem. 73, 417-435 (2004).

2. Gregoretti, I. V., Lee, Y. M. \& Goodson, H. V. Molecular evolution of the histone deacetylase family: functional implications of phylogenetic analysis. J. Mol. Biol. 338, 17-31 (2004). An insightful phylogenetic analysis of HDACs.

3. Leipe, D. D. \& Landsman, D. Histone deacetylases, acetoin utilization proteins and acetylpolyamine amidohydrolases are members of an ancient protein superfamily. Nucleic Acids Res. 25, 3693-3997 (1997).

4. Kouzarides, T. Acetylation: a regulatory modification to rival phosphorylation? EMBO J. 19, 1176-1179 (2000).

5. Richmond, T. J. \& Davey, C. A. The structure of DNA in the nucleosome core. Nature 423, 145-150 (2003).

6. Strahl, B. D. \& Allis, C. D. The language of covalent histone modifications. Nature 403, 41-45 (2000).

7. Turner, B. M. Cellular memory and the histone code. Cell 111, 285-291 (2002).

References 6 and 7 contain the proposal and one of the counter-proposals of the histone code.

8. Turner, B. M. Reading signals on the nucleosome with a new nomenclature for modified histones. Nature Struct. Mol. Biol. 12, 110-112 (2005).

9. Allfrey, V. G., Faulkner, R. \& Mirsky, A. E. Acetylation and methylation of histones and their possible role in the regulation of RNA synthesis. Proc. Natl Acad. Sci. USA 51, 786-794 (1964).

10. Polo, S. E. \& Almouzni, G. Histone metabolic pathways and chromatin assembly factors as proliferation markers. Cancer Lett. 220, 1-9 (2005).

11. Vidanes, G. M., Bonilla, C. Y. \& Toczyski, D. P. Complicated tails: histone modifications and the DNA damage response. Cell 121, 973-976 (2005).

12. Weinreich, M., Palacios DeBeer, M. A. \& Fox, C. A. The activities of eukaryotic replication origins in chromatin. Biochim. Biophys. Acta 1677, 142-157 (2004).

13. Zhang, J., Xu, F., Hashimshony, T., Keshet, I. \& Cedar, H. Establishment of transcriptional competence in early and late S phase. Nature 420, 198-202 (2002).

14. Mann, M. \& Jensen, O. N. Proteomic analysis of posttranslational modifications. Nature Biotechnol. 21, 255-261 (2003)

15. Qiang, L., Xiao, H., Campos, E. I., Ho, V. C. \& Li, G. Development of a PAN-specific, affinity-purified antiacetylated lysine antibody for detection, identification, isolation, and intracellular localization of acetylated protein. J. Immunoassay Immunochem. 26, 13-23 (2005).

16. Ronzoni, S., Faretta, M., Ballarini, M., Pelicci, P. \& Minucci, S. New method to detect histone acetylation levels by flow cytometry. Cytometry A 66A, 52-61 (2005).

17. Caron, C., Boyault, C. \& Khochbin, S. Regulatory cross-talk between lysine acetylation and ubiquitination: role in the control of protein stability. Bioessays 27, 408-415 (2005).

18. Giandomenico, V., Simonsson, M., Grönroos, E. \& Ericsson, J. Coactivator-dependent acetylation stabilizes members of the SREBP family of transcription factors. Mol. Cell. Biol. 23, 2587-2599 (2003).

19. Grönroos, E., Hellman, U., Heldin, C. H. \& Ericsson, J. Control of Smad7 stability by competition between acetylation and ubiquitination. Mol. Cell 10 483-493 (2002)

20. Jin, Y. H. et al. Transforming growth factor- $\beta$ stimulates p300-dependent RUNX3 acetylation, which inhibits ubiquitination-mediated degradation. J. Biol. Chem 279, 29409-29417 (2004)

21. Rausa, F. M., Hughes, D. E. \& Costa, R. H. Stability of the hepatocyte nuclear factor 6 transcription factor requires acetylation by the CREB-binding protein coactivator. J. Biol. Chem. 279, 43070-43076 (2004).

22. Munshi, $\mathrm{N}$ et al. Coordination of a transcriptional switch by HMGI(Y) acetylation. Science 293 1133-1136 (2001)

23. Yuan, Z. L., Guan, Y. J., Chatterjee, D. \& Chin, Y. E. Stat3 dimerization regulated by reversible acetylation of a single lysine residue. Science 307, 269-273 (2005).

24. Wang, R., Cherukuri, P. \& Luo, J. Activation of Stat3 sequence-specific DNA binding and transcription by p300/CREB-binding protein-mediated acetylation. J. Biol. Chem. 280, 11528-11534 (2005).
25. Jeong, J. W. et al. Regulation and destabilization of HIF- $1 \alpha$ by ARD 1 -mediated acetylation. Cell 111 , 709-7020 (2002)

26. Bilton, R. et al. Arrest-defective-1 protein, an acetyltransferase, does not alter stability of hypoxiainducible factor (HIF)- $1 \alpha$ and is not induced by hypoxia or HIF. J. Biol. Chem. 280, 31132-31140 (2005)

27. Cohen HY, et al. Acetylation of the $\mathrm{C}$ terminus of Ku70 by CBP and PCAF controls Bax-mediated apoptosis. Mol. Cell 13, 627-638 (2004). Cytoplasmic role for acetylation of Ku70 in apoptosis.

28. Cohen, H. Y. et al. Calorie restriction promotes mammalian cell survival by inducing the SIRT 1 deacetylase. Science 305, 390-392 (2004).

29. Bonaldi, T. et al. Monocytic cells hyperacetylate chromatin protein HMGB1 to redirect it towards secretion. EMBO J. 22, 5551-5560 (2003).

30. Bannister, A. J., Miska, E. A. Görlich, D \& Kouzarides, T. Acetylation of importin- $\alpha$ nuclear import factors by CBP/p300. Curr. Biol. 10, 467-470 (2000)

31. Caillaud, A. et al. Acetylation of interferon regulatory factor- 7 by $\mathrm{p} 300 / \mathrm{CREB}-$ binding protein (CBP)associated factor (PCAF) impairs its DNA binding. J. Biol. Chem. 277, 49417-49421 (2002).

32. Choi, C. H., Hiromura, M. \& Usheva, A. Transcription factor IIB acetylates itself to regulate transcription. Nature 424, 965-969 (2003).

33. Hasan, S. et al. Acetylation regulates the DNA end trimming activity of DNA polymerase $\beta$. Mol. Cell 10 1213-1222 (2002)

34. Hasan, S. et al. Regulation of human flap endonuclease- 1 activity by acetylation through the transcriptional coactivator p300. Mol. Cell 7 , 1221-1231 (2001)

35. Bode, A. M. \& Dong, Z. Post-translational modification of p53 in tumorigenesis. Nature Rev. Cancer 4, 793-805 (2004)

36. Chen, L. F. \& Greene, W. C. Shaping the nuclear action of NF-кB. Nature Rev. Mol. Cell Biol. 5, 392-401 (2004).

37. Kawaguchi, Y. et al. The deacetylase HDAC6 regulates aggresome formation and cell viability in response to misfolded protein stress. Cell 115, 727-738 (2003).

38. Kovacs, J. J. et al. HDAC6 regulates Hsp90 acetylation and chaperone-dependent activation of glucocorticoid receptor. Mol. Cell 18, 601-607 (2005).

Role of acetylation in the control of the chaperone function of HSP9O.

39. Lagger, G. et al. Essential function of histone deacetylase 1 in proliferation control and CDK inhibitor repression. EMBO J. 21, 2672-2681 (2002).

Genetic demonstration that HDAC1 is essential for proliferation in mammals.

40. Zhang, C. L. et al. Class II histone deacetylases act as signal-responsive repressors of cardiac hypertrophy. Cell 110, 479-488 (2002)

41. Vega, R. B. et al. Histone deacetylase 4 controls chondrocyte hypertrophy during skeletogenesis. Cell 119, 555-566 (2004)

42. Minucci, S., Nervi, C., Lo Coco, F. \& Pelicci, P. G. Histone deacetylases: a common molecular target for differentiation treatment of acute myeloid leukemias? Oncogene 20, 3110-3115 (2001)

43. Warrell, R. P. et al. Differentiation therapy of acute promyelocytic leukemia with tretinoin (all-transretinoic acid). N. Engl. J. Med. 324, 1385-1393 (1991).

44. Minucci, S. \& Pelicci, P. G. Retinoid receptors in health and disease: co-regulators and the chromatin connection. Semin. Cell Dev. Biol. 10, 215-225 (1999).

45. Lin, R. J., Egan, D. A. \& Evans, R. M. Molecular genetics of acute promyelocytic leukemia. Trends Genet. 15, 179-184 (1999)

46. Lin, R. J. \& Evans, R. M. Acquisition of oncogenic potential by RAR chimeras in acute promyelocytic leukemia through formation of homodimers. Mol. Cell 5, 821-830 (2000)

47. Minucci, S. et al. Oligomerization of RAR and AML1 transcription factors as a novel mechanism of oncogenic activation. Mol. Cell 5, 811-820 (2000).

48. Di Croce, L. et al. Altered epigenetic signals in human disease. Cancer Biol. Ther. 3, 831-837 (2004).

49. Di Croce, L. et al. Methyltransferase recruitment and DNA hypermethylation of target promoters by an oncogenic transcription factor. Science 295 1079-1082 (2002)
50. He, L. Z. et al. Histone deacetylase inhibitors induce remission in transgenic models of therapy-resistant acute promyelocytic leukemia. J. Clin. Invest. 108, 1321-1330 (2001).

51. Brown, D. et al. A PMLRAR $\alpha$ transgene initiates murine acute promyelocytic leukemia. Proc. Natl Acad. Sci. USA 94, 2551-2556 (1997).

52. Grisolano, J. L., Wesselschmidt, R. L., Pelicci, P. G. \& Ley, T. J. Altered myeloid development and acute leukemia in transgenic mice expressing PML-RAR $\alpha$ under control of cathepsin $\mathrm{G}$ regulatory sequences. Blood 89, 376-387 (1997).

53. Trecca, D. et al. Analysis of $p 53$ gene mutations in acute myeloid leukemia. Am. J. Hematol. 46, 304-309 (1994)

54. Insinga, A., Pelicci, P. G. \& Minucci, S. Leukemiaassociated fusion proteins. Multiple mechanisms of action to drive cell transformation. Cell Cycle 4 67-69 (2005)

55. Insinga, A. et al. Impairment of p53 acetylation, stability and function by an oncogenic transcription factor. EMBO J. 23, 1144-1154 (2004). Demonstrates that deacetylation of non-histone substrates (p53) by HDACs has a role in tumorigenesis.

56. Bereshchenko, O. R., Gu, W. \& Dalla-Favera, R. Acetylation inactivates the transcriptional repressor BCL6. Nature Genet. 32, 606-613 (2002).

57. Eckner, R., Arany, Z., Ewen, M., Sellers, W. \& Livingston, D. M. The adenovirus E1 A-associated 300-kD protein exhibits properties of a transcriptional coactivator and belongs to an evolutionarily conserved family. Cold Spring Harb. Symp. Quant. Biol. 59, 85-95 (1994)

58. Iyer, N. G., Ozdag, H. \& Caldas, C. p300/CBP and cancer. Oncogene 23, 4225-4231 (2004).

59. Kumar, R., Wang, R. A. \& Bagheri-Yarmand, R. Emerging roles of MTA family members in human cancers. Semin. Oncol. 30, 30-37 (2003).

60. Bagheri-Yarmand, R., Talukder, A. H., Wang, R. A Vadlamudi, R. K. \& Kumar, R. Metastasis-associated protein 1 deregulation causes inappropriate mammary gland development and tumorigenesis. Development 131, 3469-3479 (2004).

61 Zhu, P. et al. Induction of HDAC2 expression upon loss of APC in colorectal tumorigenesis. Cancer Cell $\mathbf{5}$, 455-463 (2004)

62. Toh, Y. et al. Expression of the metastasis-associated MTA1 protein and its relationship to deacetylation of the histone $\mathrm{H} 4$ in esophageal squamous cell carcinomas. Int. J. Cancer 110, 362-367 (2004).

63. Fraga, M. F. et al. Loss of acetylation at Lys 16 and trimethylation at Lys 20 of histone $\mathrm{H} 4$ is a common hallmark of human cancer. Nature Genet. 37, 391-400 (2005)

References 62 and 63 demonstrate that global histone modifications occur in cancer.

64. Seligson, D. B. et al. Global histone modification patterns predict risk of prostate cancer recurrence. Nature 435, 1262-1266 (2005).

A coordinated pattern of histone modifications predicts clinical outcome in prostate cancer.

65. Egger, G., Liang, G., Aparicio, A. \& Jones, P. A. Epigenetics in human disease and prospects for epigenetic therapy. Nature 429, 457-463 (2004).

66. Feinberg, A. P. \& Tycko, B. The history of cancer epigenetics. Nature Rev. Cancer 4, 143-153 (2004).

67. Lund, A. H. \& van Lohuizen, M. Epigenetics and cancer. Genes Dev. 18, 2315-2335 (2004).

68. Johnstone, R. W. Histone-deacetylase inhibitors: nove drugs for the treatment of cancer. Nature Rev. Drug Discov. 1, 287-299 (2002)

69. Marks, P. et al. Histone deacetylases and cancer: causes and therapies. Nature Rev. Cancer 1, 194-202 (2001)

70. Mai, A. et al. Histone deacetylation in epigenetics: an attractive target for anticancer therapy. Med. Res. Rev. 25, 261-309 (2005)

71. Miller, T. A., Witter, D. J. \& Belvedere, S. Histone deacetylase inhibitors. J. Med. Chem. 46 5097-5116 (2003)

72. Park, J. H. et al. Class I histone deacetylase-selective novel synthetic inhibitors potently inhibit human tumor proliferation. Clin. Cancer Res. 10, 5271-5281 (2004).

73. Mai, A. et al. Discovery of (aryloxopropenyl)pyrrolyl hydroxyamides as selective inhibitors of class Ila histone deacetylase homologue HD1-A. J. Med. Chem. 46, 4826-4829 (2003).

74. Heltweg, B. et al. Subtype selective substrates for histone deacetylases. J. Med. Chem. 47, 5235-5243 (2004). 
75. Haggarty, S. J., Koeller, K. M., Wong, J. C., Grozinger, C. M. \& Schreiber, S. L. Domain-selective smallmolecule inhibitor of histone deacetylase 6 (HDAC6)mediated tubulin deacetylation. Proc. Natl Acad. Sci. USA 100, 4389-4394 (2003).

76. Finnin, M. S. et al. Structures of a histone deacetylase homologue bound to the TSA and SAHA inhibitors. Nature 401, 188-193 (1999).

77. Richon, V. M. et al. Histone deacetylase inhibitors: assays to assess effectiveness in vitro and in vivo. Methods Enzymol. 376, 199-205 (2004).

78. Somoza, J. R. et al. Structural snapshots of human HDAC8 provide insights into the class I histone deacetylases. Structure (Camb) 12, 1325-1334 (2004).

79. Vannini, A. et al. Crystal structure of a eukaryotic zincdependent histone deacetylase, human HDAC8, complexed with a hydroxamic acid inhibitor. Proc. Natl Acad. Sci. USA 101, 15064-15069 (2004).

References 78 and 79 show the structural analysis of mammalian HDAC8 in complex with HDACi.

80. Siddiqui, H., Solomon, D. A., Gunawardena, R. W. Wang, Y. \& Knudsen, E. S. Histone deacetylation of RB-responsive promoters: requisite for specific gene repression but dispensable for cell cycle inhibition. Mol. Cell. Biol. 23, 7719-7731 (2003).

81. Glaser, K. B. et al. Gene expression profiling of multiple histone deacetylase (HDAC) inhibitors: defining a common gene set produced by HDAC inhibition in T24 and MDA carcinoma cell lines. $\mathrm{Mol}$. Cancer Ther. 2, 151-163 (2003)

82. Mitsiades, C. S. et al. Transcriptional signature of histone deacetylase inhibition in multiple myeloma: biological and clinical implications. Proc. Natl Acad. Sci. USA 101, 540-545 (2004).

83 Peart, M. J. et al. Identification and functional significance of genes regulated by structurally different histone deacetylase inhibitors. Proc. Natl Acad. Sci. USA 102, 3697-3702 (2005).

84. Van Lint, C., Emiliani, S. \& Verdin, E. The expression of a small fraction of cellular genes is changed in response to histone hyperacetylation. Gene Expr. 5 245-253 (1996)

References 81-84 detail gene-profiling studies of the cell transcriptional response to $\mathrm{HDACi}$ in different model systems. The results are not always fully consistent among the different systems (the number of modulated genes varies from $<5 \%$ to $20 \%$ ).

85. Camphausen, K. et al. Enhanced radiation-induced cell killing and prolongation of $\gamma \mathrm{H} 2 \mathrm{AX}$ foci expression by the histone deacetylase inhibitor MS-275. Cancer Res. 64, 316-321 (2004).

86. Munshi, A. et al. Histone deacetylase inhibitors radiosensitize human melanoma cells by suppressing DNA repair activity. Clin. Cancer Res. 11, 4912-4922 (2005).

87. Warrener, R. et al. Tumor cell-selective cytotoxicity by targeting cell cycle checkpoints. FASEB J. 17 1550-1552 (2003).

88. Beamish, H., Warrener, R. \& Gabrielli, B. G. Analysis of checkpoint responses to histone deacetylase inhibitors. Methods Mol. Biol. 281, 245-259 (2004)

89. Qiu, L. et al. Histone deacetylase inhibitors trigger a G2 checkpoint in normal cells that is defective in tumor cells. Mol. Biol. Cell 11, 2069-2083 (2000)

90. Richon, V. M., Sandhoff, T. W., Rifkind, R. A. \& Marks, P. A. Histone deacetylase inhibitor selectively induces p21WAF1 expression and gene-associated histone acetylation. Proc. Natl Acad. Sci. USA 97, 10014-10019 (2000)

91. Gui, C. Y., Ngo, L., Xu, W. S, Richon, V. M. \& Marks, P. A Histone deacetylase (HDAC) inhibitor activation of p21WAF1 involves changes in promoter-associated proteins, including HDAC1. Proc. Natl Acad. Sci. USA $101,1241-1246$ (2004)

92. Varshochi, R. et al. ICI182,780 induces $p 21$ Waf gene transcription through releasing histone deacetylase 1 and estrogen receptor $\alpha$ from $\mathrm{Sp} 1$ sites to induce cell cycle arrest in MCF-7 breast cancer cell line. J. Biol. Chem. 280, 3185-3196 (2005).

93. Archer, S. Y., Meng, S., Shei, A. \& Hodin, R. A p21(WAF1) is required for butyrate-mediated growth inhibition of human colon cancer cells. Proc. Natl Acad. Sci. USA 95, 6791-6796 (1998).

94. Shao, Y., Gao, Z., Marks, P. A. \& Jiang, X. Apoptotic and autophagic cell death induced by histone deacetylase inhibitors. Proc. Natl Acad. Sci. USA 101 18030-18035 (2004)

95. Rosato, R. R., Almenara, J. A., Grant, S. The histone deacetylase inhibitor MS-275 promotes differentiation or apoptosis in human leukemia cells through a process regulated by generation of reactive oxygen species and induction of p21CIP1/WAF1 1 Cancer Res. 63, 3637-3645 (2003).

96. Ungerstedt, J. S. et al. Role of thioredoxin in the response of normal and transformed cells to histone deacetylase inhibitors. Proc. Natl Acad. Sci. USA 102 673-678 (2005)

97. Subramanian, C., Opipari, A. W., Bian, X., Castle, V. P. $\&$ Kwok, R. P. Ku 70 acetylation mediates neuroblastoma cell death induced by histone deacetylase inhibitors. Proc. Natl Acad. Sci. USA 102 4842-4847 (2005)

98. Atadja, P. et al. Molecular and cellular basis for the anti-proliferative effects of the HDAC inhibito LAQ824. Novartis Found. Symp. 259, 249-266 (2004)

99. Bali, P. et al. Inhibition of histone deacetylase 6 acetylates and disrupts the chaperone function of heat shock protein 90: a novel basis for antileukemia activity of histone deacetylase inhibitors. J. Biol. Chem 280, 26729-26734 (2005).

100. Hideshima, T. et al. Small-molecule inhibition of proteasome and aggresome function induces synergistic antitumor activity in multiple myeloma. Proc. Natl Acad. Sci. USA 102, 8567-8572 (2005).

101. Insinga, A. et al. Inhibitors of histone deacetylases induce tumor-selective apoptosis through activation of the death receptor pathway. Nature Med. 11, 71-76 (2005)

102. Nebbioso, A. et al. Tumor-selective action of HDAC inhibitors involves TRAIL induction in acute myeloid leukemia cells. Nature Med. 11, 77-84 (2005). References 101 and 102 demonstrate the essentia role of induction of the TRAIL pathway for the proapoptotic response following HDACi treatment in leukaemias.

103. Inoue, H. et al. Histone deacetylase inhibitors sensitize human colonic adenocarcinoma cell lines to TNF-related apoptosis inducing ligand-mediated apoptosis. Int. J. Mol. Med. 9, 521-525 (2002).

104. Nakata, S. et al. Histone deacetylase inhibitors upregulate death receptor 5/TRAIL-R2 and sensitize apoptosis induced by TRAIL/APO2-L in human malignant tumor cells. Oncogene 23, 6261-6271 (2004).

105. Singh, T. R., Shankar, S. \& Srivastava, R. K. HDAC inhibitors enhance the apoptosis-inducing potential of TRAIL in breast carcinoma. Oncogene $\mathbf{2 4}$ 4609-4623 (2005)

106. Watanabe, K., Okamoto, K. \& Yonehara, S. Sensitization of osteosarcoma cells to death receptormediated apoptosis by HDAC inhibitors through downregulation of cellular FLIP. Cell Death Differ. 12 10-18 (2005)

107. Rosato, R. R. \& Grant, S. Histone deacetylase inhibitors in clinical development. Expert Opin Investig. Drugs 13, 21-38 (2004)

108. Johnstone, R. W. \& Licht, J. D. Histone deacetylase inhibitors in cancer therapy: is transcription the primary target? Cancer Cell 4, 13-18 (2003)

109. Drummond, D. C. et al. Clinical development of histone deacetylase inhibitors as anticancer agents. Annu. Rev. Pharmacol. Toxicol. 45, 495-528 (2004)

110. Chavez-Blanco, A. et al. Histone acetylation and histone deacetylase activity of magnesium valproate in tumor and peripheral blood of patients with cervical cancer. A phase I study. Mol. Cancer 4, 22 (2005).

111. Kelly, W. K. et al. Phase I study of an oral histone deacetylase inhibitor, suberoylanilide hydroxamic acid, in patients with advanced cancer. J. Clin. Oncol. 23, 3923-3931 (2005)

112. Kelly, W. K. et al. Phase I clinical trial of histone deacetylase inhibitor: suberoylanilide hydroxamic acid administered intravenously. Clin. Cancer Res. 9 3578-3588 (2003)

113. Ryan, Q. C. et al. Phase I and pharmacokinetic study of MS-275, a histone deacetylase inhibitor, in patients with advanced and refractory solid tumors or lymphoma. J. Clin. Oncol. 23, 3912-3922 (2005).

114. Piekarz, R. L. et al. Inhibitor of histone deacetylation, depsipeptide (FR901228), in the treatment of peripheral and cutaneous T-cell lymphoma: a case report. Blood 98, 2865-2868 (2001).

115. Sandor, V. et al. Phase I trial of the histone deacetylase inhibitor, depsipeptide (FR901228, NSC 630176), in patients with refractory neoplasms. Clin. Cancer Res. 8, 718-728 (2002).

116. Piekarz, R. L. et al. T-cell lymphoma as a model for the use of histone deacetylase inhibitors in cancer therapy: impact of depsipeptide on molecular markers, therapeutic targets, and mechanisms of resistance. Blood 103, 4636-4643 (2004).

117. Bandyopadhyay, D., Mishra, A. \& Medrano, E. E. Overexpression of histone deacetylase 1 confers resistance to sodium butyrate-mediated apoptosis in melanoma cells through a p53-mediated pathway. Cancer Res. 64, 7706-7710 (2004).

118. Xiao, J. J. et al. Chemoresistance to depsipeptide FK228 [(E)-(1S,4S, 10S,21R)-7-[(Z)-ethylidene]-4,21diisopropyl-2-oxa-12,13-dithia-5,8,20,23 tetraazabicyclo[8,7,6]-tricos-16-ene-3,6,9,22 pentanone] is mediated by reversible MDR1 induction in human cancer cell lines. J. Pharmacol. Exp. Ther. 314, 467-475 (2005).

119. Pilatrino, C. et al. Increase in platelet count in older, poor-risk patients with acute myeloid leukemia or myelodysplastic syndrome treated with valproic acid and all-trans retinoic acid. Cancer 104, 101-109 (2005).

120. Cameron, E. E., Bachman, K. E., Myöhänen, S., Herman, J. G. \& Baylin, S. B. Synergy of demethylation and histone deacetylase inhibition in the re-expression of genes silenced in cancer. Nature Genet. 21, 103-107 (1999).

121. Rahmani, M. et al. Cotreatment with suberanoylanilide hydroxamic acid and 17-allylamino 17-demethoxygeldanamycin synergistically induces apoptosis in $\mathrm{Bcr}-\mathrm{Abl}{ }^{+}$cells sensitive and resistant to STI571 (imatinib mesylate) in association with downregulation of $\mathrm{Bcr}-\mathrm{Abl}$, abrogation of signal transduce and activator of transcription 5 activity, and Bax conformational change. Mol. Pharmacol. 67 , 1166-1176 (2005)

122. Girdwood, D. W., Tatham, M. H. \& Hay, R. T. SUMO and transcriptional regulation. Semin. Cell Dev. Biol. 15, 201-210 (2004)

123. Al-Hajj, M., Becker, M. W., Wicha, M., Weissman, I. \& Clarke, M. F. Therapeutic implications of cancer stem cells. Curr. Opin. Genet. Dev. 14, 43-47 (2004).

124. Reya, T., Morrison, S. J., Clarke, M. F. \& Weissman, I. L. Stem cells, cancer, and cancer stem cells. Nature $\mathbf{4 1 4}$ 105-111 (2001)

125. Milhem, M. et al. Modification of hematopoietic stem cell fate by 5 aza 2'deoxycytidine and trichostatin A. Blood 103, 4102-4110 (2004). An initial study on the effects of HDACi on stem cells.

126. Guenther, M. G. et al. A core SMRT corepressor complex containing HDAC3 and TBL1, a WD40-repeat protein linked to deafness. Genes Dev. 14 1048-1057 (2000)

127. Huang, E. Y. et al. Nuclear receptor corepressors partner with class II histone deacetylases in a Sin3. independent repression pathway. Genes Dev. 14 45-54 (2000).

128. Humphrey, G. W. et al. Stable histone deacetylase complexes distinguished by the presence of SANT domain proteins CoREST/kiaa0071 and Mta-L1. J. Biol. Chem. 276, 6817-6824 (2001).

29. Jones, P. L., Sachs, L. M., Rouse, N., Wade, P. A. \& Shi, $\mathrm{Y}$. B Multiple $\mathrm{N}$-CoR complexes contain distinct histone deacetylases. J. Biol. Chem. 276 8807-8811 (2001)

130. Li, J. et al. Both corepressor proteins SMRT and $\mathrm{N}$-CoR exist in large protein complexes containing HDAC3. EMBO J. 19, 4342-4350 (2000).

131. Xue, Y. et al. NURD, a novel complex with both ATPdependent chromatin-remodeling and histone deacetylase activities. Mol. Cell 2, 851-861 (1998)

132. Yao, Y. L. \& Yang, W. M. The metastasis-associated proteins 1 and 2 form distinct protein complexes with histone deacetylase activity. J. Biol. Chem. 278, 42560-42568 (2003).

133. Zhang, Y. et al. Analysis of the NuRD subunits reveals a histone deacetylase core complex and a connection with DNA methylation. Genes Dev. 13, 1924-1935 (1999).

134. Seigneurin-Berny, D. et al. Identification of components of the murine histone deacetylase 6 complex: link between acetylation and ubiquitination signaling pathways. Mol. Cell. Biol. 21, 8035-8044 (2001).

135. Yamagoe, S. et al. Interaction of histone acetylases and deacetylases in vivo. Mol. Cell. Biol. 23 1025-1033 (2003).

136. Fischle, W. et al. Enzymatic activity associated with class II HDACs is dependent on a multiprotein complex containing HDAC3 and SMRT/N-CoR. Mol. Cell 9, 45-57 (2002)

137 Verdin, E., Dequiedt, F \& Kasler, H. G. Class II histone deacetylases: versatile regulators. Trends Genet. 19 286-293 (2003) 
138. Gore, S. D. et al. Impact of prolonged infusions of the putative differentiating agent sodium phenylbutyrate on myelodysplastic syndromes and acute myeloid leukemia. Clin. Cancer Res. 8, 963-970 (2002)

139. Patnaik, A. et al. A phase I study of pivaloyloxymethyl butyrate, a prodrug of the differentiating agent butyric acid, in patients with advanced solid malignancies. Clin. Cancer Res. 8, 2142-2148 (2002).

140. Raffoux, E., Chaibi, P., Dombret, H. \& Degos, L. Valproic acid and all-trans retinoic acid for the treatment of elderly patients with acute myeloid leukemia. Haematologica 90, 986-988 (2005).

141. Byrd, J. C. et al. A phase 1 and pharmacodynamic study of depsipeptide (FK228) in chronic lymphocytic leukemia and acute myeloid leukemia. Blood 105 959-967 (2005)

Acknowledgements

We acknowledge Bruno Amati, Gordon McVie, Antonello Mai, Michela Prudenziati, Roberta Carbone Jr, Gabriele Bucci, Oronzina Botrugno, Maurizio Moroni, members of our laboratories for discussions and critical reading of the manuscript, and the reviewers of this manuscript for their insightful comments.

\section{Competing interests statement}

The authors declare competing financial interests: see web version for details.
DATABASES

The following terms in this article are linked online to Entrez Gene: http://www.ncbi.nlm.nih.gov/entrez/query. fcgi?db=gene

BAX |CBP | DR5 |FAS |FASL | HDAC1 | HDAC2 | HDAC4 | HDAC5 | HDAC6 | HDAC9 | histone H4 | Ku70|MTA1 | p21 | p27 | p53 | p300|PML | PLZF | RAR | RB | STAT3 | TRAIL | TXN National Cancer Institute: http://www.cancer.gov Acute promyelocytic leukaemia

SUPPLEMENTARY INFORMATION

See online article: S1 (table)

Access to this links box is available online. 